\title{
Evaluation of defense strategy against Influenza A in cell line
}

\section{models [version 1; peer review: 2 approved with reservations]}

\author{
Ekaterina Antonova(D1, Olga Glazova1, Anna Gaponova1, Aykaz Eremyan (iD1, \\ Natalya Grebenkina1, Svetlana Zvereva1, Natalya Volkova², Pavel Volchkov
}

${ }^{1}$ Moscow Institute of Physics and Technology, Dolgoprudny, Moscow Region, 141701, Russian Federation
${ }^{2}$ Ernst Institute of Animal Husbandry, Podolsk Municipal District, Moscow Region, 142132, Russian Federation

V1 First published: 19 Feb 2018, 7:206

https://doi.org/10.12688/f1000research.13496.1

Latest published: 16 May 2018, 7:206

https://doi.org/10.12688/f1000research.13496.2

\section{Abstract}

Background: Influenza virus can cause both seasonal infections and unpredictable pandemics. Rapidly evolving avian H5N1 virus is getting increasingly infective for humans. Since avian Influenza can be transmitted by domestic birds, serving as a key link between wild aquatic birds and humans, an effective measure to control the influenza transmission would be eradication of the infection in poultry. It is known that the virus penetrates into the cell through binding with the terminal oligosaccharides - sialic acids (SA) - on the cell surfaces. Removal of SA might be a potential antiviral strategy. An approach to developing chicken lines that are resistant to influenza viruses could be the creation of genetically modified birds. Thus it is necessary to select a gene that provides defense to influenza. Here we have expressed in cells a range of exogenous sialidases and estimated their activity and specificity towards SA residues.

Methods: Several bacterial, viral and human sialidases were tested. We adopted bacterial sialidases from Salmonella and Actinomyces for expression on the cell surface by fusing catalytic domains with transmembrane domains. We also selected Influenza A/PuertoRico/8/34/H1N1 neuraminidase and human membrane sialidase ( $h N e u 3$ ) genes. Lectin binding assay was used for estimation of a a $(2,3)$-sialylation level by fluorescent microscopy and FACS.

Results: We compared sialidases from bacteria, Influenza virus and human. Sialidases from Salmonella and Influenza A neuraminidase effectively cleaved a (2-3)-SA receptors. Viral neuraminidase demonstrated a higher activity. Sialidases from Actinomyces and $h N e u 3$ did not show any activity against a (2-3) SA under physiological conditions.

Conclusion: Our results demonstrated that sialidases with different specificity and activity can be selected as genes providing antiviral defence. Combining chosen sialidases with different activity together with tissue-specific promoters would provide an optimal level of desialilation to prevent infection. Tissue specific expression of the sialidases could protect domestic birds from infection.

\section{Open Peer Review \\ Approval Status \\ 1 \\ 2 \\ version 2 \\ (revision) \\ 16 May 2018 \\ version 1 \\ 19 Feb 2018

view
$?$
view

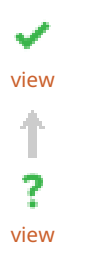 \\ 1. Gillian M Air, University of Oklahoma Health \\ Sciences Center, Oklahoma City, USA \\ 2. Laurence S. Tiley, University of Cambridge, \\ Cambridge, UK}

Any reports and responses or comments on the article can be found at the end of the article. 
Keywords

sialidases, Influenza A, defence strategy, exogenous expression, $a(2$,

3)-sialylation

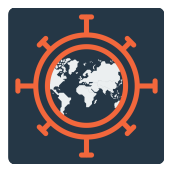

This article is included in the Emerging Diseases

and Outbreaks gateway.

Corresponding authors: Ekaterina Antonova (enryabkova@gmail.com), Pavel Volchkov (vpwwww@gmail.com)

Author roles: Antonova E: Formal Analysis, Funding Acquisition, Investigation, Writing - Original Draft Preparation; Glazova O:

Validation, Visualization; Gaponova A: Methodology; Eremyan A: Software; Grebenkina N: Resources; Zvereva S: Resources; Volkova N

: Data Curation; Volchkov P: Conceptualization, Data Curation, Project Administration, Writing - Review \& Editing

Competing interests: No competing interests were disclosed.

Grant information: This work has completed with the financial support of Russian Science Foundation within project No. 16-16-04094.

Copyright: @ 2018 Antonova $\mathrm{E}$ et al. This is an open access article distributed under the terms of the Creative Commons Attribution License, which permits unrestricted use, distribution, and reproduction in any medium, provided the original work is properly cited.

How to cite this article: Antonova E, Glazova O, Gaponova A et al. Evaluation of defense strategy against Influenza A in cell line models [version 1; peer review: 2 approved with reservations] F1000Research 2018, 7:206

https://doi.org/10.12688/f1000research.13496.1

First published: 19 Feb 2018, 7:206 https://doi.org/10.12688/f1000research.13496.1 


\section{Introduction}

The global influenza pandemic remains a real threat. Avian influenza viruses H5N1 have killed millions of birds, including domestic poultry, causing enormous financial losses. Several thousands of cases of human infection were recorded and some with a fatal outcome (see World Health Organisation (WHO) assessment of Avian Flu pandemic threat). The risk of a new pandemic of viral infection remains high. Seasonal flu vaccination is used as a traditional way to prevent the infection spread, however it has a lot of limitations. Therefore, it is critical to develop alternative approaches to prevent influenza infection. An alternative way to reduce the infection could be the creation of genetically modified domestic birds. It will decrease the risk of the infection spread, because domestic birds are known to transmit the infection acting as an intermediary between wild ducks to humans (Kim et al., 2009). It is necessary to select genecandidates for developing the approach.

The best way to prevent the infection is to impede the virus from entering the cell. Influenza virus hemagglutinin (HA) provides attachment to the host cell that leads to fusion between the virion envelope and the host cell membrane (Skehel \& Wiley, 2000). Surface sialic acid (SA) residues are host cell epitopes that are recognized by influenza virus A and B (Ito, 2000). SA are a family of nine-carbon acidic monosaccharides that naturally terminate sugar chains attached to the proteins on the cell surface (Varki et al., 2009). Neuraminidase (NA) - the second major surface antigen - is an exoglycosidase, or saildase, that cleaves SA from cell membrane glycolipids and glycoproteins, thus destroying the recognition epitopes on the surface of the host cell for the viral receptor HA. NA activity helps viral particles penetrate through mucous secretions that are rich in sialic acids, to reach the target cells in the airway epithelium (Palese et al., 1974). The role of the enzyme in facilitating release of newly formed viral particles from the infected cell surface and preventing aggregation of the viral particles was experimentally confirmed (Matrosovich et al., 2004; Palese et al., 1974). There are known antiviral agents - oseltamivir and zanamivir - that inhibit NA, blocking the release of virus particles from infected cells (Moscona, 2005; Varghese, 1999). Sialidases have also demonstrated to be effective inhibitors of Influenza virus infection in vitro. It has been shown that cells treated with Vibrio cholerae or Micromonospora viridifaciens bacterial sialidase are resistant to influenza virus infection (Air \& Laver, 1995; Bergelson et al., 1982; Griffin et al., 1983; Stray et al., 2000).

We focused on exogenous expression of sialidases as a defense strategy against influenza infection (Figure 1). Here we show the protective effect of exogenous expression of different sialidases. The range of exogenous sialidases has diverse activity and specificity towards sialic acid residues. Tissue-specific expression of sialidases in transgenic poultry might protect domestic birds against Influenza virus.
A

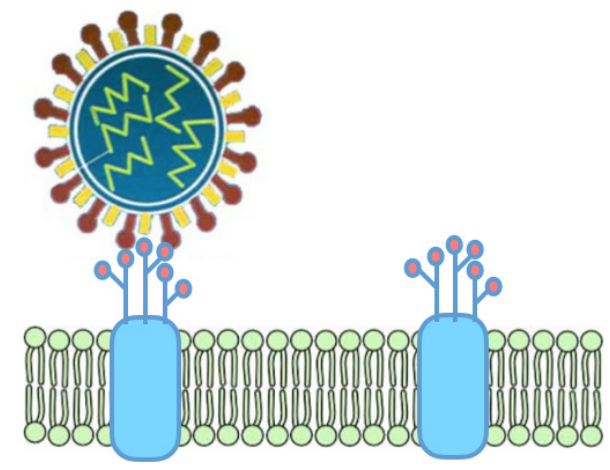

hemagglutinin of

Influenza A virus recognises sialic acids
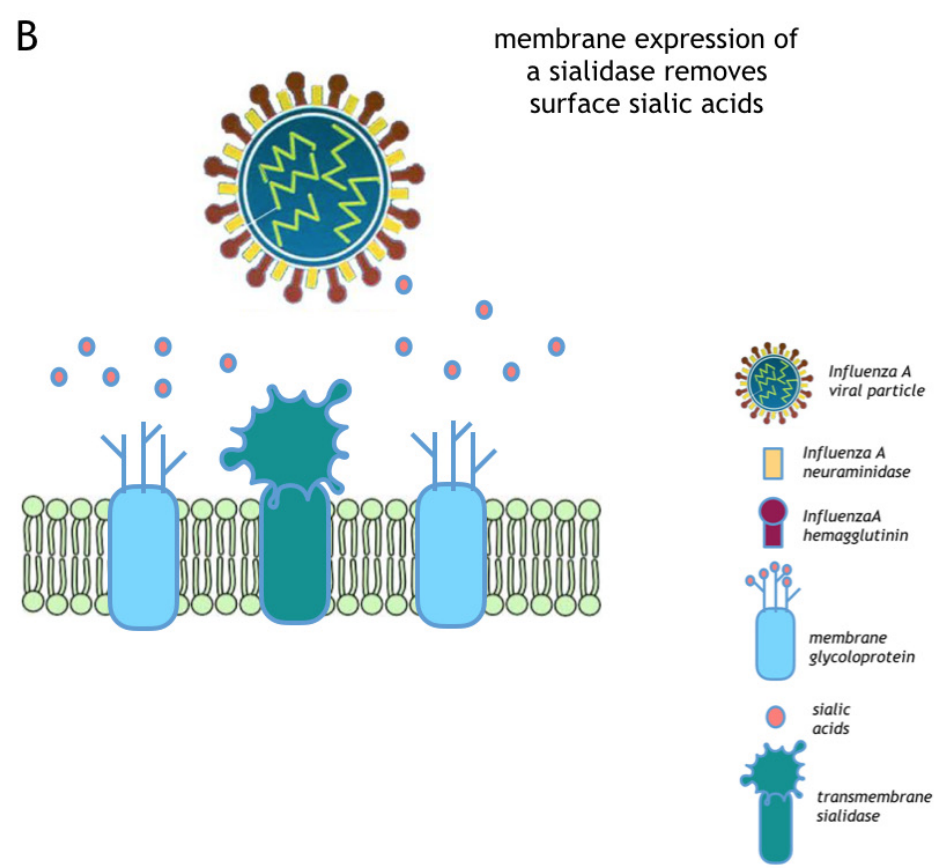

and virus penetrates in the host cell

\section{virus doesn't penetrate} in the cell

Figure 1. Schematic of the antiviral strategy. (A) Influenza A virus penetrates into the host cell through the binding with the host cell surface sialic acids. (B) Removal of sialic acids through exogenous sialidase expression protects cells from viral infection. 


\section{Methods}

\section{Cloning of sialidases}

Viral neuraminidase. Viral neuraminidase from human Influenza A virus was amplified from the plasmid pNAHA (Plasmid \# 44169; Addgene, Cambridge, MA, USA) that encodes the neuraminidase gene of Influenza A / Puerto Rico / 8/34, subtype N1. The amplified product was inserted by restriction cloning in the lentiviral transfer plasmid based on a pHAGE backbone (\#24526, Addgene) after the CMV promoter. The RFP reporter was cloned in the same reading frame and the resulted construction was pHAGE-CMV-infNA-P2A-RFP (Supplementary Figure 1A).

\section{Bacterial sialidases}

Sialidases from Salmonella typhimurium and Actinomyces viscosus. Synthesis of the codon-optimized bacterial sialidase from Salmonella typhimurium (Uniprot / P29768, 2-382aa) and the codon-optimized bacterial sialidase from Actinomyces viscosus (uniprot / Q59164, 347-631aa) fused with the transmembrane domain of viral neuraminidase (1 - 75 aa) were done in BioCat. The transmembrane domain was added to provide membrane localisation of the enzymes, since sialidase Salmonella.t. and A. viscosus are cytoplasmic. The codonoptimization was done with the IDT tool with the choice of the genome of Gallus gallus. The genes were ordered in the standard vector pUC57 and were flanked by the restriction sites NheI and SphI for S. typhimurium and XhoI and SphI sites for A. viscosus. The genes were excised from the vector pUC57 using the sites and inserted into the pHAGE backbone after the the CMV promoter. As a result, the plasmids pHAGE-CMV-Sal.t.SiaP2A-RFP and pHAGE-CMV-Act.v.Sia-P2A-RFP have been cloned (Supplementary Figure 1A).

\section{Human neuraminidase}

The genes coding sialidase 3 (membrane sialidase) in G.gallus and H.sapiens are homologous based on the analysis in HomoloGene (NCBI). We selected human membrane sialidase (Gene ID: 10825) for cloning because there is more information about it compared with other orthologs. The coding exons were amplified from human genomic DNA and overlapped. The resulted product was inserted by restriction cloning in the lentiviral transfer plasmid based on the pHAGE backbone (\#24526, Addgene) after CMV promoter. The RFP reporter was cloned in the same reading frame and the resulted construction was pHAGE-CMV-hNeu3-P2A-RFP (Supplementary Figure 1A).

\section{Catalytically inactive sialidase}

Enzymatically inactive neuraminidase was taken as a negative control. Two amino acids at positions 262-263 (EE) of the Influenza A virus strain A/Puerto Rico/8/1934 H1N1 are responsible for the substrate binding. The inactive variant can be obtained by the mutation E262D (Huang et al., 2008). The mutation was made by two overlapping primers: 5'-GCACCTAATTCTCACTATG AtGAATGTTCCTGTTAC-3', 5'-CATTCaTCATAGTGAGAAT TAGGTGC-3' that change the codon from GAG to GAT (The changed nucleotides for the overlapping primers are marked as lowercase letters). Primers were made by Evrogen (Moscow, Russia). The changed PCR product was then cloned into the same backbone.
Tet-on system, design and cloning

Tet-on system was set up to make transcription of the gene of interest to be inducible (Gossen \& Bujard, 1992; Gossen et al., 1995). rtTA (tetracycline transactivator), CMV promoter, TRE (Tet Response Element) promoter P2A and RFP were amplified from different plasmids. Influenza A neuraminidase and sialidase from S. typhimurium were inserted under control of TRE-promoter. Neuraminidase was amplified from the plasmid pNAHA (Plasmid \# 44169, Addgene) and sialidase S. typhimurium was amplified from pHAGE-CMV-Sal.t.Sia-RFP. All amplified products were cloned in the pHAGE backbone in the following order: TRE-infNA/Sal.t.Sia-P2A-RFP-CMV-rtta by restriction cloning (Supplementary Figure 1B). The plasmid pTagBFP encoding BFP (blue fluorescent protein) was used for cotransfection to approximately estimate effectiveness of transfection before addition of the doxycycline.

\section{Doxycycline induction}

Cells were treated with Doxycycline (D9891, Sigma; St Louis, MI, USA) at 24 hours after transfection at a concentration of $0.5 \mu \mathrm{g} / \mathrm{ml}$. Expression of the RFP reporter and activity of genes-enzymes were analyzed at 48 hours after Doxycycline addition.

\section{Cell cultivation, transfection, microscopy and FACS analysis}

MDCK and HEK293 cell lines were cultured according to ATCC recommendations. Cells were maintained at $37^{\circ} \mathrm{C}$ with $5 \% \mathrm{CO}_{2}$. Cells were transfected with TurboFect reagent (R0531, Thermo Fisher; Waltham, MA, USA) according to the manufacturer recommendations. The fluorescence microscope Axio observer (Zeiss, Oberkochen, Germany) with the standard set of filters was used for visualization of fluorescence. S3e BioRad sorter (Hercules, CA, USA) was used for fluorescence activated cell sorting and flow cytometry. Standard flow cytometry analysis was carried out using FlowJo v10.4 software.

\section{Statistical analysis}

FITC fluorescence in Lectin binding assay was measured by FACS and quantified as an average mean fluorescence intensity (MFI). Values show the means \pm SD of triplicate results from representative experiments. Three independent experiments were carried out for each experimental case. Student's $t$-test was used to determine the level of statistical significance. Statistical analysis was perform in Microsoft Excel 2016

\section{Lentiviral production, determination of viral titre and viral transduction of MDCK cell line}

Lentivirus packaging was performed according to the described protocol [see Protocol 1: Lentivirus Packaging by 293T Transfection from CReM].

In order to determine a viral titre, different aliquots of supernatant were added to HEK293 cells in the presence of $4 \mu \mathrm{g} / \mathrm{ml}$ polybrene (Hexadimethrine bromide, H9268, Sigma). Infection of six 10-fold serial dilutions was performed in 96-well plates. The medium was replaced 12 hours after infection. Three serial infections were performed. At 48 hours after infection, transduction effectiveness was calculated by FACS analysis. The viral titer 
corresponded to the number of colonies developed at the highest dilution.

The supernatant of the known titre was used for viral transduction of the MDCK cell line. On the first day about $1.0 \times 10-4$ MDCK cells were seeded into 6-well plates. The cells were incubated $18-20$ hours at $37^{\circ} \mathrm{C}$ in a humidified incubator in an atmosphere of 5-7\% CO2. Upon transduction the confluency around 30-50\% was estimated. Next, the appropriate volumes of unconcentrated virus and polybrene with concentration $5 \mathrm{ug} / \mathrm{ml}$ were added to each well. After 48 hours, cells were analyzed using Flow cytometry to define the percentage of infected/fluorescent cells. Lentiviral transduction efficiency was calculated as (the number of RFP-positive cells/the total number of cells counted $\times 100$ ) and the number of transduced cells (transduction efficiency $(\%) \times$ the total number of RFP recovered/100).

\section{Genomic DNA extraction and PCR analysis}

Genomic DNA was isolated using Wizard ${ }^{\circledR}$ Genomic DNA Purification Kit (A1120, Promega, Madison, WI, USA) according the manufacturer protocol.

\section{Lectin binding assay}

Lectin binding assay was used for the cell surface SA detection. For lectin binding assay we used Maackia amurensis (MA) lectins that are specific for $\alpha$ (2-3)-bound SA. The lectin were conjugated with the FITC fluorophore (21761036-1 (510183), bio-WORLD; Dublin, OH, USA). The assay was made for HEK293 and MDCK cells. The experiment was setup in 48-well plates. The cells expressing a desired construct were washed three times with PBS. Then cells were incubated with MA lectins (1:100) about 1 hour and three times washed with PBS. Hoechst (RRID:AB_2651133, Thermo Fisher) was added for visualization of the nuclei for $5 \mathrm{~min}$ at recommended concentration with the subsequent washing with PBS.

\section{Results}

Transient expression of genetic constructs in HEK293 cell lines

Sialidases vary in enzyme kinetic and substrate specificity to the type of the SA linkage. Several sialidases from bacteria, Influenza virus and vertebrate were selected for our study. We were mostly interested in $\alpha(2-3)$ specialized sialidases, because avian Influenza prefers this type of linkage (Ito, 2000). However the broad substrate specificity was also an area of interest. Regarding enzyme kinetics, sialidases with various levels of activity were useful for us in order to combine them with tissue-specific promoters and find an optimal level of desialilation. The optimal sialic acids level on the cells surface prevents penetration of the virus into the cell and does not affect cell function.

Substrate specificity of bacterial sialidases is very diverse, in terms of type of sialic acid linkage and enzyme kinetics. We selected two different bacterial sialidases: Salmonella typhimurium sialidase, which specializes in cleavage of $\alpha(2-3)$ sialic acid residues (Hoyer et al., 1991; Rogerieux et al., 1993) and Actinomyces viscosus sialidase, which has a substrate specificity to both $\alpha(2-3)$ and $\alpha$ (2-6), but preferentially cleaves the $\alpha$ (2-6) linkage (Teufel et al., 1989). We used sequence of the catalytic domains of the sialidases and added to their $\mathrm{N}$-terminal sequences the transmembrane domain with the stem loop of Influenza NA transmembrane protein in order to target the proteins to the membrane and demonstrate its catalytic activity outside of the cell.

The human Influenza neuraminidase (sialidase) has been taken for analysis as a broad-spectrum sialidase. Unlike human HA that preferentially recognises $\alpha(2-6)$, the viral NA of $\mathrm{H} 1 \mathrm{~N} 1$ has cleavage activity to both (2-6) and $\alpha(2-3)$ types of linkages.

Exogenous overexpression of a vertebrate membrane sialidase also would be interesting to analyze. The sialidases are poorly investigated, but some experimental data exists for human membrane neuraminidase hNeu3 (Monti et al., 2000; Zhang et al., 2010) and the enzyme was selected for cloning.

All cloned genetic constructs were firstly tested by transient expression in the HEK293 cell line that has glycosylation patterns including $\alpha$ (2-3) and $\alpha$ (2-6) sialic acid linkages (Picanco-Castro et al., 2013). All plasmids had the RFP reporter to mark the cells that express a sialidase. Using lectin binding assays it has been shown that the cells expressing the sialidase from $S$. typhimurium have decreased level of SA. Expression of the sialidase from A. viscosus did not affect the $\alpha$ (2-3) sialylation level, probably because it is more specific to the $\alpha(2,6)$ sialic acid linkages. However, it has previously been shown that this sialidase effectively removes both $\alpha(2,3)$ - and $\alpha$ (2,6)-linked SA from the cell surface (Malakhov et al., 2006). Human neuraminidase hNEU3 also has not shown activity towards $\alpha$ (2-3)-linked SA. We suppose that it might be related to unsuitable $\mathrm{pH}$ conditions. Cultivation in DMEM in $5 \% \mathrm{CO}_{2}$ provides $\mathrm{pH}$ in range of 7,6-7,7. Earlier, hNeu3 activity was demonstrated in the $\mathrm{pH}$ range of 2.8-6.6 (Monti et al., 2000). In the case of influenza A neuraminidase, sialylation level decreased, not only in RFP-positive cells, but also in RFP-negative cells, which are not supposed to express the enzyme (Figure 2A). According to the flow cytometry analysis, the most significant $\alpha$ (2-3)-linked SA cleavage was in the case of Influenza A neuraminidase expression (Figure 2B). Representative histograms from three independent repeats are shown. Lectin binding assay was quantified as fluorescence and calculated as a mean fluorescence intensity - MFI $( \pm S D)$ : d_infNA-RFP 103.437 ( \pm 3.568$)$, Act.v.Sia-RFP - 181.401( \pm 7.988$)$, Sal. t.Sia-RFP - 7.341( \pm 1.323$)$, infNA-RFP - 4.725( \pm 983$)$, hNeu3 $27.379( \pm 2.231)$.

\section{Inducible expression of genetic constructs in HEK293 cell} lines

A responsive transcription of a sialidase gene gives an opportunity to induce the gene expression in response to a molecule addition. In our case we used Tetracycline-Controlled Transcriptional Activation where transcription is reversibly turned on in the presence of the antibiotic tetracycline derivative doxycycline. The method can be a useful model for studying viral infection 
A

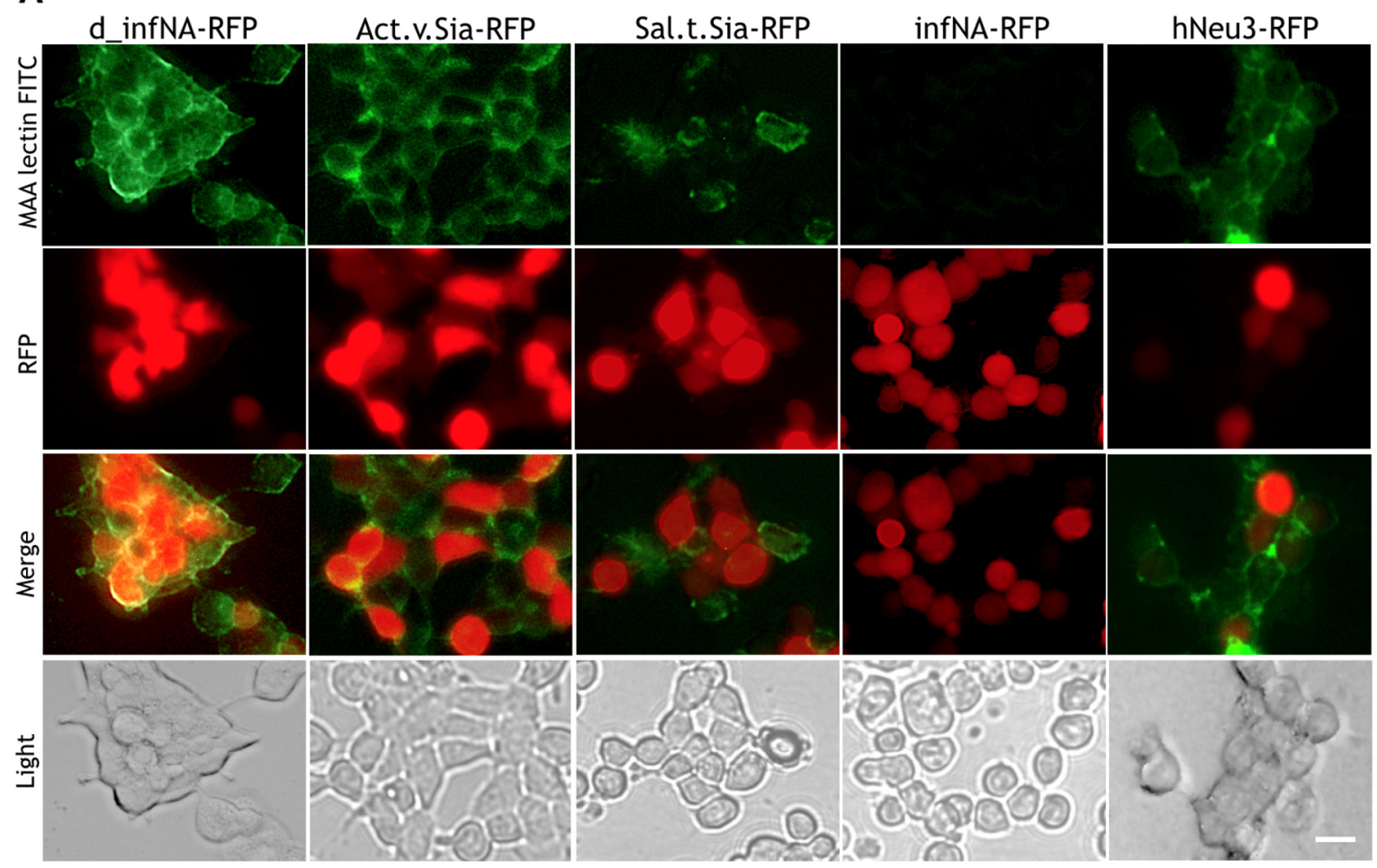

B
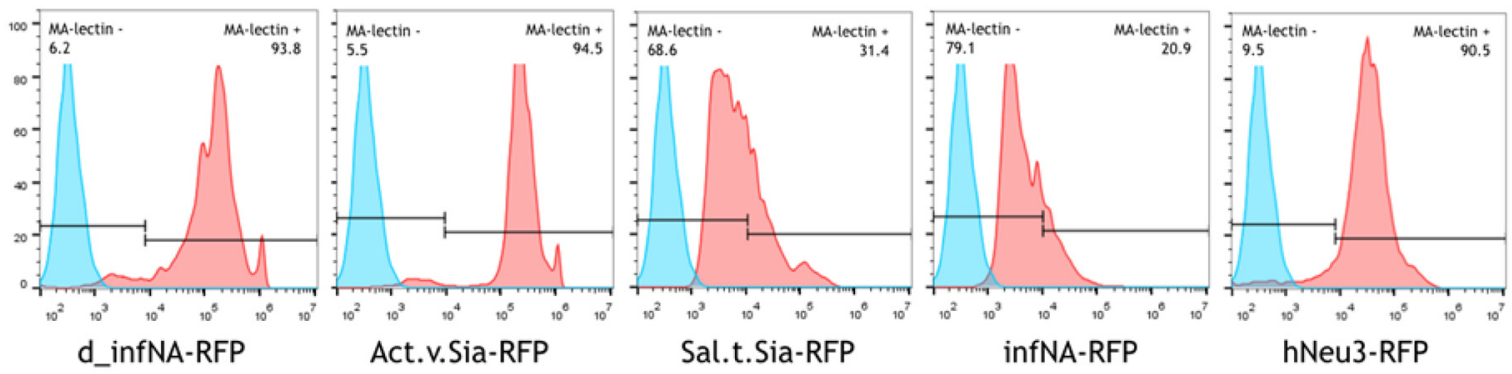

Figure 2. Evaluation of sialidase activity in the HEK293 cell line. Genetic constructs were transiently expressed in the HEK293 cell line, and sialidase activity was evaluated using a lectin binding assay with FITC-labelled Maackia amurensis lectins. The plasmid with catalytically inactive neuraminidase was used as the negative control. (A) Fluorescent microscopy analysis. Scale bar $=10$ um. (B) FACS analysis.

in cell culture and especially for use in genetically modified organisms because constitutive exogenous gene expression could have a potential harm.

For tetracycline-inducible expression we selected only sialidases that demonstrated significant $\alpha(2-3)$ catalytic activity in the previous experiment with transient expression under CMV promoter (infNA-RFP and Sal.t.Sia-RFP). The plasmid coding BFP under constitutive CMV promoter was used for estimation of transfection effectiveness. It was shown that upon treatment of cells with doxycycline the sialidases expression switched on and as a result the $\alpha(2-3)$ sialylation level decreased (Figure 3A, B).
From flow cytometry analysis representative histograms from three repeats are shown. MFI $( \pm \mathrm{SD})$ was calculated: infNARFP, +dox - $1.468( \pm 439)$, Sal.t.Sia-RFP, +dox - 6.437( \pm 1.043$)$, d_infNA-RFP, + dox $-83.849( \pm 2.983)$.

\section{Lentiviral transduction of MDCK cell lines}

The MDCK cell line is used for propagation of influenza viruses. To obtain cell lines that stably express exogenous genes coding for different sialidases we transduced MDCK cells with lentiviruses. The expressing construct was integrated into the genome by lentiviral transduction. The integration was confirmed with PCR (Supplementary Figures 2 and 3) using 

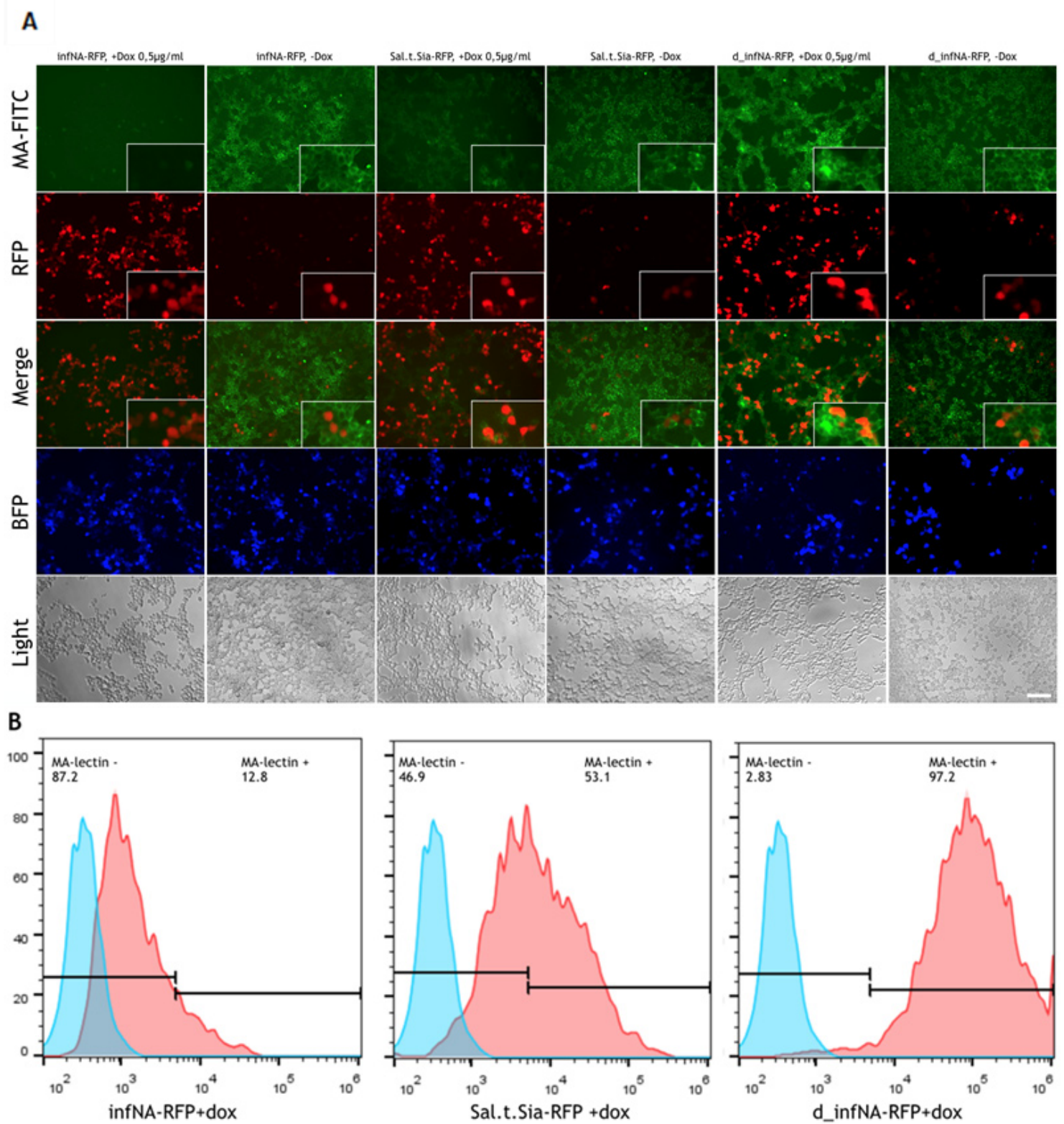

Figure 3. Doxycycline-inducible expression of S. typhimurium sialidase and Influenza A neuraminidase in the HEK293 cell line. Gene expression was evaluated in the HEK293 cell line after doxycycline induction, using a lectin binding assay with FITC-labelled Maackia amurensis lectins. The plasmid with catalytically inactive neuraminidase was used as the negative control. (A) The plasmid coding BFP was used to estimate the effectiveness of transfection. Fluorescent microscopy analysis. Scale bar $=50$ um. (B) FACS analysis.

primers from Table 1. The effect of expression of sialidases was studied by testing the reactivity of cells with sialic acid linkage-specific lectins.

The results confirmed the previously obtained data with the HEK293 cell line. The cells expressing S. typhimyrium sialidase and Influenza A neuraminidase had decreased level of the surface $\alpha$ (2-3) SA. However, the decrease in the SA level in the MDCK was less significant compared with the HEK293 cell line (Figure 4B). Representative histograms from three technical repeats are shown. MFI was calculated: d_infNA-RFP - 38.120( \pm 2.322$)$, Act.v.Sia-RFP - 22.750( \pm 3.175$)$, Sal.t.Sia-RFP - 21.732( \pm 4.598$)$,
infNA-RFP - 7.290( \pm 1.934$)$, RFP-NA - 12.798( \pm 3.453$)$. Similarly with the experiments on the HEK293 cell line, in the case of infNA-RFP expression $\alpha$ (2-3) SA were absent not only in the RFP-positive cells but in the RFP-negative cells as well. A possible explanation of the evidence could be a 'slipstream' translocation in a polycistronic vector of the $\mathrm{P} 2 \mathrm{~A}$ downstream protein without a signal of localization that is RFP in our case (de Felipe et al., 2010). An alternatively explanation being when a ribosome encounters $2 \mathrm{~A}$ within an open reading frame the synthesis of a specific peptide bond could be "skipped". This results in termination of translation at the end of $2 \mathrm{~A}$ peptide (Donnelly et al., 2001). It means that RFP is not expressed and can explain 
Table 1. Primers used to confirm the presence of exogenous insertion.

\begin{tabular}{l|l|l|}
$\begin{array}{l}\text { Name of } \\
\text { insertion }\end{array}$ & Forward primer & Reverse primer \\
\hline Sal.t.Sia-RFP & 5'-AGCGCCACCATGAACCCGAACC-3' & 5'-CAATTAAGTTTGTGCCCCAGTTTGC-3' \\
\hline Act.v.Sia-RFP & 5'GGATCCGCTAGCCGCCACCATGAAC-3' & 5'-CAATTAAGTTTGTGCCCCAGTTTGC-3' \\
\hline infNA-RFP & 5'-TGGGCTATATACAGCAAAGAC-3' & 5'-CAATTAAGTTTGTGCCCCAGTTTGC-3' \\
\hline RFP-NA & 5'-CATGGTGTCTAAGGGCGAAGAGC-3' & 5'-CGCCTACTTGTCAATGCTGAATGGCAAC-3' \\
\hline RFP & 5'-CATGGTGTCTAAGGGCGAAGAGC-3' & 5'-CAATTAAGTTTGTGCCCCAGTTTGC-3' \\
\hline
\end{tabular}

A

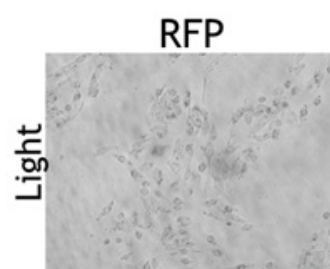

Act.v.Sia-RFP

Sal.t.Sia-RFP

infNA-RFP
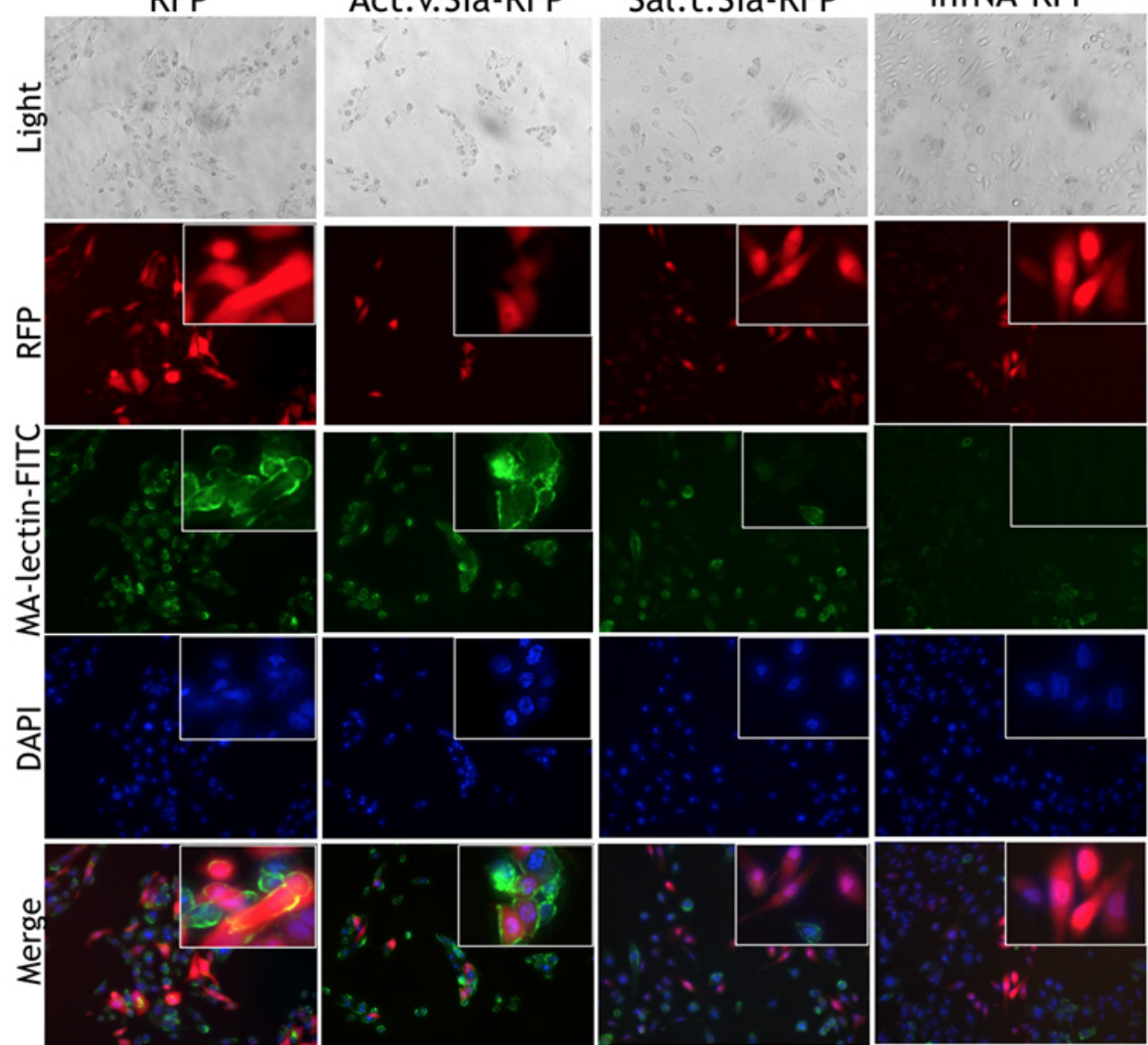

B
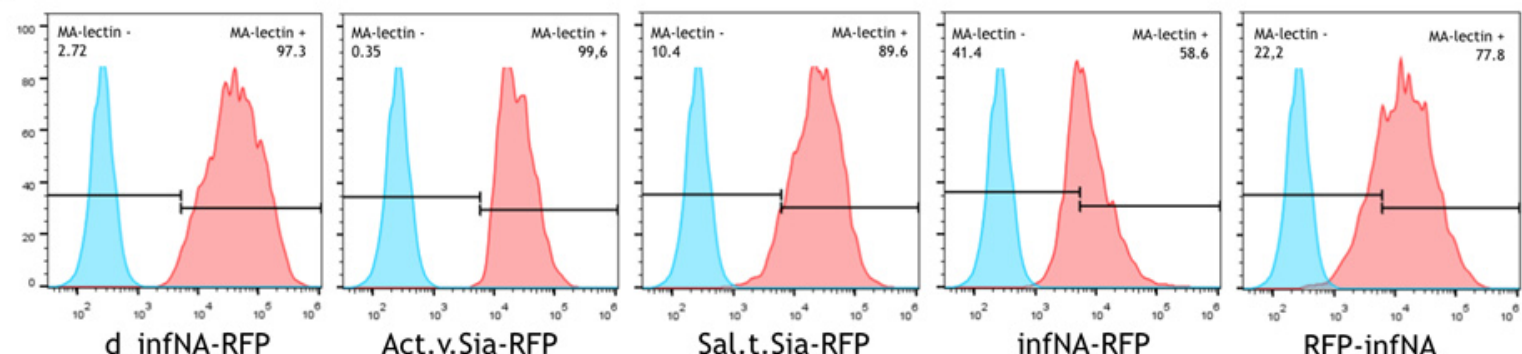

Figure 4. Evaluation of sialidase activity in the MDCK cell line. MDCK cells were transduced with lentiviruses. Lectin binding assay was made with FITC-labelled Maackia amurensis lectins. The plasmid with catalytically inactive neuraminidase was used as the negative control. (A) Fluorescent microscopy analysis. Scale bar $=50$ um. (B) FACS analysis. 
the absence of sialic acids in some RFP-negative cells. The effects were not observed in the case of other sialidases. Usage of a longer sequence for P2A (with a favorable upstream sequence composition) or modifying the order of proteins could solve the problem. When we changed the order of the RFP and the infNA sequences (RFP-P2A-infNA), the result of lectin binding assay was as expected: RFP-positive cells showed decreased level of $\alpha$ (2-3) SA (Figure 4A). Nevertheless, in the case of inverted position of the neuraminidase the enzyme activity was less pronounced. It can be explained by the fact that a protein located at the second position can be slightly less expressed in a polycistronic construct with a 2A peptide (Liu et al., 2017). In the case of the $\mathrm{S}$. typhimyrium sialidase expression (Sal.t.Sia-RFP) the 'slipstream' effect was not observed.

\section{Discussion}

Sialic acid is the sole receptor of Influenza A virus (Matrosovich et al., 2013). Therefore the removal of sialic acids from the cell surface might be a powerful defence strategy against Influenza virus. In the current study we take a variety of sialidases from different sources and compared their activity. We demonstrated that expression of the sialidase catalytic domain from Salmonella typhimurium fused with the transmembrane domain and human Influenza A neuraminidase effectively removes $\alpha$ (2-3)-sialic residues on the cell surface under physiological conditions.

In our study, about $70-80 \%$ of the surface SA were removed by the membrane sialidases. It has been reported that the virus binding could still occur with such sialylation level, as has been shown in the MDCK cell line, but amplification of Influenza virus was still inhibited (Stray et al., 2000), therefore complete elimination of SA is perhaps unnecessary. Neuraminidase had higher cleavage activity against $\alpha(2-3)$-linked sialic acids than sialidase from $\mathrm{S}$. typhimyrium according to our results. An optimal level of desialilation can be established in the tissue when combining genetic constructs with varying sialidase activity and tissue-specific promoters.
Stable expression of sialidases potentially could disrupt physiological functions required for proper glycosylation (Gutierrez et al., 1987; Michalek et al., 1988; Pangburn et al., 2000; Varki, 1992). Inducible expression may be more suitable in this case. The Tet-on inducible system allowed robust expression and functioning of genetic constructs encoding sialidases after the inductor addition. The level of sialilation will return to the previous level during a period of time after withdrawal of doxycycline. The required time depends on the sialic acid turnover rate.

It has been shown that sialidase treatment does not affect the properties of respiratory mucus, nor did it affect the normal mucus transport activity on ciliated epithelium (King et al., 1974; Meyer et al., 1975). Thus, temporary desialilation of the epithelial surface should not cause problems.

Our current preliminary in vitro data indicates that sialidases from Salmonella typhimurium and neuraminidase from Influenza A virus could be the potential candidates that provide antiviral defense against avian Influenza virus. Since sialidases target cellular receptors but not a viral gene product, the chance of influenza viruses developing resistance is low.

\section{Data availability}

Data underlying this study is available from Dataverse - doi: 10.7910/DVN/UCDPX3 (Antonova, 2018).

\section{Competing interests}

No competing interests were disclosed.

\section{Grant information}

This work has completed with the financial support of Russian Science Foundation within project No. 16-16-04094.
Air GM, Laver WG: Red cells bound to influenza virus N9 neuraminidase are not released by the N9 neuraminidase activity. Virology. 1995; 211(1): 278-284. PubMed Abstract | Publisher Full Text

Antonova E: Evaluation of defence strategy against Influenza $A$ in cell line models. Harvard Dataverse, V1 2018. Publisher Full Text

Bergelson LD, Bukrinskaya AG, Prokazova NV, et al:: Role of gangliosides in reception of influenza virus. Eur J Biochem. 1982; 128(2-3): 467-474. PubMed Abstract | Publisher Full Text

de Felipe P, Luke GA, Brown JD, et al.: Inhibition of 2A-mediated 'cleavage' of certain artificial polyproteins bearing $\mathrm{N}$-terminal signal sequences. Biotechno J. 2010; 5(2): 213-223.

PubMed Abstract | Publisher Full Text | Free Full Text

Donnelly ML, Luke G, Mehrotra A, et al:: Analysis of the aphthovirus 2A/2B polyprotein 'cleavage' mechanism indicates not a proteolytic reaction, but a novel translational effect: a putative ribosomal 'skip'. J Gen Virol. 2001;
82(Pt 5): 1013-25

PubMed Abstract | Publisher Full Text

Gossen $\mathrm{M}$, Bujard $\mathrm{H}$ : Tight control of gene expression in mammalian cells by tetracycline-responsive promoters. Proc Natl Acad Sci U S A. 1992; 89(12): 5547-51.

PubMed Abstract | Publisher Full Text | Free Full Text

Gossen M, Freundlieb S, Bender G, et al.: Transcriptional activation by tetracyclines in mammalian cells. Science. 1995; 268(5218): 1766-9. PubMed Abstract | Publisher Full Text

Griffin JA, Basak S, Compans RW: Effects of hexose starvation and the role of sialic acid in influenza virus release. Virology. 1983; 125(2): 324-334. PubMed Abstract | Publisher Full Text

Gutierrez C, Martin MJ, Brown KA: Complement activation by human lymphocytes from different lymphoid organs: role of sialic acid and lack of relationship to electrical surface charge. Complement. 1987; 4(2): 99-109. PubMed Abstract | Publisher Full Text 
Hoyer LL, Roggentin P, Schauer R, et al.: Purification and properties of cloned Salmonella typhimurium LT2 sialidase with virus-typical kinetic preference for sialyl alpha 2----3 linkages. J Biochem. 1991; 110(3): 462-7.

PubMed Abstract | Publisher Full Text

Huang IC, Li W, Sui J, et al.: Influenza A virus neuraminidase limits viral superinfection. J Virol. 2008; 82(10): 4834-4843.

PubMed Abstract | Publisher Full Text | Free Full Text

Ito $\mathrm{T}$ : Interspecies transmission and receptor recognition of influenza A viruses. Microbiol Immunol. 2000; 44(6): 423-30.

PubMed Abstract | Publisher Full Text

Kim JK, Negovetich NJ, Forrest HL, et al:: Ducks: The "Trojan Horses" of H5N1

influenza. Influenza Other Respir Viruses. 2009; 3(4): 121-128.

PubMed Abstract | Publisher Full Text | Free Full Text

King M, Gilboa A, Meyer FA, et al:: On the transport of mucus and its rheologic simulants in ciliated systems. Am Rev Respir Dis. 1974; 110(6): 740-5. PubMed Abstract

Liu Z, Chen O, Wall JBJ, et al.: Systematic comparison of 2A peptides for cloning multi-genes in a polycistronic vector. Sci Rep. 2017; 7(1):

2193.

PubMed Abstract | Publisher Full Text | Free Full Text

Matrosovich M, Herrler G, Klenk HD: Sialic Acid Receptors of Viruses. Top Curr Chem. 2013; 367: 1-28

PubMed Abstract | Publisher Full Text

Matrosovich MN, Matrosovich TY, Gray T, et al: Human and avian influenza viruses target different cell types in cultures of human airway epithelium. Proc Natl Acad Sci U S A. 2004; 101(13): 4620-4.

PubMed Abstract | Publisher Full Text | Free Full Text

Malakhov MP, Aschenbrenner LM, Smee DF, et al: Sialidase Fusion Protein as a Novel Broad-Spectrum Inhibitor of Influenza Virus Infection. Antimicrob Agents Chemother. 2006; 50(4): 1470-1479.

PubMed Abstract | Publisher Full Text | Free Full Text

Meyer FA, King M, Gelman RA: On the role of sialic acid in the rheological properties of mucus. Biochim Biophys Acta. 1975; 392(2): 223-32.

PubMed Abstract | Publisher Full Text

Michalek MT, Bremer EG, Mold C: Effect of gangliosides on activation of the alternative pathway of human complement. J Immunol. 1988; 140(5):

$1581-7$

PubMed Abstract

Monti E, Bassi MT, Papini N, et al:: Identification and expression of NEU3, a novel human sialidase associated to the plasma membrane. Biochem J. 2000; 349(Pt 1): 343-351.

PubMed Abstract | Publisher Full Text | Free Full Text
Moscona A: Neuraminidase inhibitors for influenza. N Engl J Med. 2005; 353(13): 1363-73.

PubMed Abstract | Publisher Full Text

Palese $\mathrm{P}$, Tobita K, Ueda M, et al:: Characterization of temperature sensitive influenza virus mutants defective in neuraminidase. Virology. 1974; 61(2):

397-410.

PubMed Abstract | Publisher Full Text

Pangburn MK, Pangburn KL, Koistinen V, et al.: Molecular mechanisms of target recognition in an innate immune system: interactions among factor $\mathrm{H}, \mathrm{C} 3 \mathrm{~b}$, and target in the alternative pathway of human complement. J Immunol. 2000 164(9): 4742-51.

PubMed Abstract | Publisher Full Text

Picanco-Castro V, Biaggio RT, Cova DT, et al:: Production of recombinant therapeutic proteins in human cells: current achievements and future perspectives. Protein Pept Lett. 2013; 20(12): 1373-81.

PubMed Abstract | Publisher Full Text

Rogerieux F, Belaise M, Terzidis-Trabelsi H, et al:: Determination of the sialic acid linkage specificity of sialidases using lectins in a solid phase assay. Anal Biochem. 1993; 211(2): 200-4.

PubMed Abstract | Publisher Full Text

Skehel JJ, Wiley DC: Receptor binding and membrane fusion in virus entry: the influenza hemagglutinin. Annu Rev Biochem. 2000; 69: 531-69.

PubMed Abstract | Publisher Full Text

Stray SJ, Cummings RD, Air GM: Influenza virus infection of desialylated cells. Glycobiology. 2000; 10(7): 649-58.

PubMed Abstract | Publisher Full Text

Teufel M, Roggentin P, Schauer R: Properties of sialidase isolated from

Actinomyces viscosus DSM 43798. Biol Chem Hoppe Seyler. 1989; 370(5): 435-43. PubMed Abstract | Publisher Full Text

Varghese JN: Development of neuraminidase inhibitors as anti-influenza virus drugs. Drug Dev Res. 1999; 46(3-4): 176-196.

Publisher Full Text

Varki A: Selectins and other mammalian sialic acid-binding lectins. Curr Opin Cell Biol. 1992; 4(2): 257-66.

PubMed Abstract | Publisher Full Text

Varki A, Cummings RD, Esko JD, et al.: Essentials of glycobiology, 2nd edn. Cold Spring Harbor Laboratory Press, New York. 2009.

PubMed Abstract

Zhang M, Koskie K, Ross JS, et al:: Enhancing glycoprotein sialylation by targeted gene silencing in mammalian cells. Biotechnol Bioeng. 2010; 105(6): 1094-1105.

PubMed Abstract | Publisher Full Text 


\section{Open Peer Review}

\section{Current Peer Review Status: ? ?}

\section{Version 1}

Reviewer Report 05 April 2018

https://doi.org/10.5256/f1000research.14654.r32149

(C) 2018 Tiley L. This is an open access peer review report distributed under the terms of the Creative Commons Attribution License, which permits unrestricted use, distribution, and reproduction in any medium, provided the original work is properly cited.

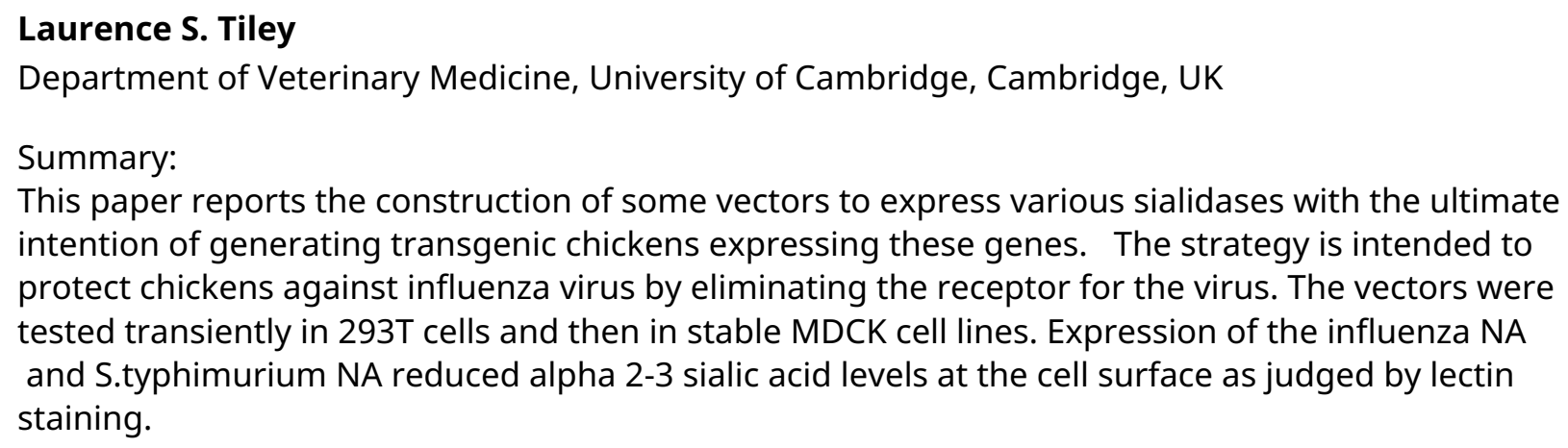
intention of generating transgenic chickens expressing these genes. The strategy is intended to protect chickens against influenza virus by eliminating the receptor for the virus. The vectors were tested transiently in 293T cells and then in stable MDCK cell lines. Expression of the influenza NA and S.typhimurium NA reduced alpha 2-3 sialic acid levels at the cell surface as judged by lectin staining.

I see very little of publishable merit at this stage of the work and the quality of the text requires substantial improvement.

Specific comments:

Abstract:

1. H5N1 is not showing increasing infection/adaptation for humans.

2. I suggest the authors consider the increased threat posed by H7N9 which is arguably of far more concern at present.

Introduction:

3. The cumulative case count for H5N1 is 454 deaths out of 860 total cases. It is unlikely that the case count of a disease with this high case fatality rate has caused "several thousand" cases and "some with fatal outcome". The wording here is very imprecise.

4. The citation is from 2005 and totally inappropriate. See:

http://www.who.int/influenza/human_animal_interface/2018_03_02_tableH5N1.pdf?ua=1

5. Seasonal flu vaccination is primarily used to protect individuals in high risk groups. Its impact on spread in the general population is probably insignificant.

Methods:

6. The construction details for RFP-P2A-infNA are not provided. 
7. The method for the determination of the lentivector titre is unclear. The analysis by FACS is OK, but what are the authors referring to when they mention "The viral titre corresponded to the number of colonies developed at the highest dilution?"

8. What exactly do they mean by "three serial infections"?

9. Typo: they used $\sim 1 \times 10^{4}$, not $1 \times 10^{-4}$ MDCK cells.

Note: I could not access all the supplementary info (even on the Harvard Dataverse).

Results:

10. d_infNA-RFP is not defined, but presumably it is the inactive E262D.

11. Surely a typo for the SD value here: infNA-RFP - 4.725( \pm 983$)$

12. Before making more complex speculations regarding the inactivity of the hNeu3 and Act.v.Sia constructs, the authors should demonstrate they are expressed in the correct compartment. RFP expression merely shows the mRNA were translated.

13. Another typo for the SD: infNA- RFP, +dox - $1.468( \pm 439)$ ?

14. The consistent apparent activity of the N1 neuraminidase acting on non-transduced cells, could more simply be an indication that the protein is being secreted. The lack of data on protein localisation does not help with the interpretation. The "slip-stream" explanation hypothesising that many more cells are transduced than were apparent in the photos is possible, but not very credible as this would also need the lentivirus titre/transduction efficiency to be much higher for the infNA vector.

15. Little consideration regarding the topology of their constructs has been given.

Discussion:

16. The discussion is quite superficial. The authors might like to mention related approaches such as DAS181 and the discussions regarding this general approach ${ }^{1}$

17. There are reports that de-sialylation does not completely abrogate influenza infectivity. A point perhaps worth mentioning briefly.

18. They may wish to mention more about the possible physiological consequences e.g. the potential impact on the MCE of constitutively expressing a sialidase ${ }^{2}$

19. The choice of appropriate promoter is not discussed in any detail. Presumably the intention is to restrict expression to relevant tissues i.e. the respiratory and GI tract in the case of poultry. Do they have a particular promoter in mind?

20. The practicalities of using a Dox-inducible promoter are not considered. Is the intention to treat flocks continuously with Dox to prevent infection, or to respond prophylactically to an outbreak in a nearby location? The use of Dox in this way is undesirable for several reasons.

\section{References}

1. Nicholls JM, Moss RB, Haslam SM: The use of sialidase therapy for respiratory viral infections. Antiviral Res. 2013; 98 (3): 401-9 PubMed Abstract | Publisher Full Text

2. Duez J, Sixt N, Pechinot A: Influenza virus infection: don't forget the role of the mucociliary 
system!. Journal of Antimicrobial Chemotherapy. 2008; 63 (2): 421-422 Publisher Full Text

Is the work clearly and accurately presented and does it cite the current literature? Partly

Is the study design appropriate and is the work technically sound?

Yes

Are sufficient details of methods and analysis provided to allow replication by others? Partly

If applicable, is the statistical analysis and its interpretation appropriate? Yes

Are all the source data underlying the results available to ensure full reproducibility? No

Are the conclusions drawn adequately supported by the results?

No

Competing Interests: No competing interests were disclosed.

Reviewer Expertise: Influenza molecular virology. Transgenic livestock.

I confirm that I have read this submission and believe that I have an appropriate level of expertise to confirm that it is of an acceptable scientific standard, however I have significant reservations, as outlined above.

Author Response 04 May 2018

Ekaterina Antonova, Moscow Institute of Physics and Technology, Dolgoprudny, Russian Federation

Abstract

1-2. Agreed. The statement regarding H5N1 was modified to 'Avian H5N1 and recently H7N9 virus has a potential pandemic threat.' in the version 2.

Introduction

3.The sentence has been corrected from "Several thousands of cases of human infection were recorded and many of them with a fatal outcome" to "860 cases of disease in human population have been detected since 2003 and 454 of them with fatal outcome (see http://www.who.int/influenza/human_animal_interface/2018_03_02_tableH5N1.pdf?ua=1

4. The reference has been changed in the version 2 .

5. Agreed. The sentence has been corrected Methods

6. The information has been added in the Methods part: "Additionally the reverse construction RFP-P2A-infNA was cloned in the same vector. (Supplementary Figure 1A)". 7. The sentence "The viral titre corresponded to the number of colonies developed at the 
highest dilution" was deleted due to it is related to the titre determination with drug selection. However lentiviral constructions with resistant genes were not considered in the publication. Instead, the following text has been added in the version 2: "FACS analysis was performed using non-infected cells as a negative control. The cells with $10 \%-40 \%$ RFPpositive were selected for titer calculation. The titer was calculated using the equation: Titer $($ Transduction Unit $/ \mathrm{ml})=($ Cell Number in each well used for infection on Day $2 \times$ percentage of GFP/RFP positive cells (should be $10 \%-40 \%$ ))/(virus volume used for infection in each well $\times$ dilution fold)"

8. The three repeats were mentioned. The phrase "three serial infections" was corrected in the text to avoid confusion: "Infection with the same aliquot was used in three repeats."

9. The typo has been corrected in the version 2.

Please check the supplementary availability now.

Supplementary Figure 1A \& 1B. Genetic constructs.

Click here to access the data.

Supplementary Figure 2. Confirmation of inserted genes in MDCK genome after lentiviral transduction.

Click here to access the data.

Supplementary Figure 3. Schematic of integrated constructs with primers for PCR analysis. Click here to access the data.

Results

10.The mention has been added in the part Methods/Catalytically inactive sialidase

11. Corrected: $4.725( \pm 0.983)$

12. We used well-known transmembrane domain of Influenza neuraminidase for proper localization of both Act.v.Sia and Sal.t.Sia sialidases on cytoplasmic membrane. The sequence of the transmembrane domain and stem loop was the same for both bacterial sialidases, however only one was active. It is known that the sialidase hNeu3 is localised on the plasma membrane. We cloned the gene and checked the sequence. Lectin binding assay was used to confirm its functionality on the cell surface hence the correct localisation (in the case if activity was demonstrated). However we agree with your point and we have noticed it in the text (Results/Transient expression of genetic constructs in HEK293 cell lines)

13. Corrected: $1.468( \pm 0.439)$

14. We have considered the hypothesis regarding secreted neuraminidase and rejected it. First, because we used neuraminidase from Influenza Puerto Rico strain (Addgene). And it is well-known that the neuraminidase is located on the plasma membrane surface. We sequenced the neuraminidase gene after cloning. Second, the reversion of positions of neuraminidase and RFP would not influenced on neuraminidase secretion. But in our case only RFP-positive cells were desialylated (CMV-RFP-P2A-infNA expression) that exclude secretion. Regarding the lentivirus titre/transduction efficiency. The lentivirus titre was defined by RFP fluorescence. However not all RFP sequences could be translated properly due to the effect of termination of translation at the end of $2 \mathrm{~A}$ peptide that resulted in lower RFP fluorescence. Hence the lentivirus titre could be underestimated. In result the cells were infected by larger quantity of viral particles. FACS data was normalised to RFP fluorescence level when analysing the level of lectin binding.

15. We used well-characterised transmembrane domain to target all the enzymes (except human Neu3) on the cell surface and made functional estimation of catalytic activity using lectin binding assay. Enzymatic activity of a protein in the cell expressing reporter confirms membrane location of the protein on the cell membrane. 
Discussion

16. The mention regarding DAS181 has been added in the discussion part: "Previously, a recombinant sialidase (DAS181) has already demonstrated its antiviral effectivity in the cell culture (Malakhov MP et al., 2006)) and in vivo, using a mouse model (intranasal injection of the recombinant fusion protein - DAS181) (Belser JA et al., 2007). Thus, the principal possibility of the usage of membrane-anchored sialidase as antiviral defence has been demonstrated."

17. We added in the discussion points regarding the possible risk: "There are several concerns to use the strategy. The first is that influenza virus receptors may be different from SA, thus, sialidase expression may be ineffective. It has been reported that the virus binding could still occur in the MDCK cell line desialylated with addition in the medium Micromonospora viridifaciens sialidase ( Stray et al., 2000). The authors suggested that influenza virus infection can result from sialic acid-independent receptors, either directly or in a multistage process. The presence of sialic acid may enhance virus binding to the cell surface to increase interaction with secondary receptors to mediate entry. Also the authors mentioned that high multiplicity of infection has an increased requirement for sialic acids and desialylation will inhibit the virus amplification. Although the HA of a human H1N1 strain was shown to bind other glycoconjugates (Rapoport et al., 2006), the exact receptors to enter into the cell have not been found. Stray et al., used NA-deficient virus in the research which was obtained in a laboratory. It is unlikely that such virus would exist in nature. Probably, the barrier to efficient infection and transmission between organisms is due to inefficient use or expression of a yet unidentified entry mediator."

18. Added: "Another concern of the stable expression of sialidases is disruption of physiological functions required for proper glycosylation ( Gutierrez et al., 1987; Michalek et al., 1988; Pangburn et al., 2000; Varki, 1992). The probable consequences of constitutive expression of membrane sialidase in vivo are not known. It has been shown that overexpression of the human ortholog NEU3 membrane sialidase under the $\beta$-actin promoter in transgenic mice resulted insulin-resistant diabetes mellitus (Sasaki et al., 2003). The authors focused on insulin signaling only. However at least the data shows that the constitutive membrane sialidase expression was not lethal for mice.

We are going to reduce a probable negative impact on organism using tissue-specific expression of the selected gene. It is necessary to predict a potential impact on the epithelium of intestine and trachea in vivo. There are no similar genetic experiments. However the consequences of tissue-specific expression could be considered from the researches where local desialylation was done. It has been shown that intranasal sialidase treatment does not affect the properties of respiratory mucus, nor did it affect the normal mucus transport activity on ciliated epithelium (King et al., 1974; Meyer et al., 1975). Some authors showed that Influenza infection is associated with an increased risk of secondary infection by Streptococcus pneumoniae (Sluijs et al., 2006). There are concerns that, probably, tissue-specific expression of sialidases would result in a greater chance of bacterial infection in the respiratory tract due to exposure of its binding sites that were masked by terminal sialic acid. It has been shown that the binding sites for the bacteria already exist on the normal respiratory surface and sialidase treatment does not introduce de novo bacterial adhesion sites. Also the risk of infection arises due to damage of mucus and epithelial cells. But it is not clear whether the secondary S. pneumoniae infection happens due to viral NA activity or from a secondary effects of influenza virus intracellular amplification. Influenza infection in the respiratory tract causes much deeper changes to the epithelium than 
treatment of the surface of the epithelial cells with a sialidase, because Influenza infection in humans and in mice results in vast exposure of the ciliated epithelial cells down to the basal cells and the basement membrane (Walsh et al , 1961; Plotkowski et al.,1986). Thus, the most likely the increased $S$. pneumoniae infection is the result of airway epithelial damage caused by the virus infection but not just desialylation.

In the gastrointestinal tract $\mathrm{SA}$ is an abundant sugar residue in mucin that is a key target of intestinal bacteria. Expression of sailidases will release free SA from mucins that could drives intestinal inflammation and infection. For example, elevated levels of free sialic acid in the gut, during and post antibiotic treatments, promote the expansion of Clostridium difficile, that utilises free SA. Also it has been shown that sialidase activity can promote the outgrowth of Escherichia coli and result in imbalanced microbiota, inflammation, colitis development due to a (2-3)-sialic residues promote expansion of $E$. coli (Huang et al., 2015). Nevertheless, we do not know the probable negative impact on the physiology of avian respiratory and digestive tract. Thus, an optimal level of desialylation can be established in the tissue when combining genetic constructs with varying sialidase activity and tissuespecific promoters to avoid disruption of physiological functions in our approach. Additionally, a gene expression with time limit could prevent a probable negative impact on a living organism. Michael P. Malakhov et al. showed that temporary desialylation of respiratory tract during 7 days using recombinant sialidase protein did not cause any symptoms of toxicity or inflammation in ferrets and mice that were not infected by the virus (Malakhov et al., 2006). This in vivo data support our idea that temporary inducible expression of a sialidase will not have serious negative physiological effects for transgenic animals."

19. Yes, we have. We have analysed several promoters specific for intestine and trachea. We have measured expression in scraping of the epithelium from the chicken intestine and trachea by RT-PCR and selected several tissue specific genes that have different expression level.

Respiratory tract: SFTPA2, SLC34A2. Related expression normalized to GAPDH: SFTPA2 $(3,5 \pm 0,7) ; \operatorname{SLC} 34 A 2(0,002) . p<0,001$

Digestive tract: RBP2, GUCA2A, MUC13, OLFM4. Related expression normalized to GAPDH: $\operatorname{RBP} 2(1,1 \pm 0,01)$;

GUCA2A (0,4 $\pm 0,005) ; \operatorname{MUC13}(0,18 \pm 0,05) ; \operatorname{OLFM} 4(0,09 \pm 0,015), p<0,01$.

20. It is planned to use Dox prophylactically to an outbreak. The practicalities of using Teton system were added: "We are planning to use the Tet-on system as a potential model for creation of genetically modified organism that allows to induce a sialidase expression temporary. Transgenic transcription factor rtTA will be expressed under control of a tissuespecific promoter and a sialidase will be expressed under control of TRE promoter. This enables tissue-specific inducible control of the transgene expression and will provide a powerful and flexible resource for studies of influence of sialylation on chicken physiology and on viral infection. The level of sialylation will return to the previous level during a period of time after withdrawal of doxycycline. The required time depends on the sialic acid turnover rate. The inducing agent can be treated prophylactically to genetically modified birds in an outbreak that can protect livestock and locally stop infection spread. It is known that the inducible system has some limitations. Firstly, it has basal transgene 'leak'. However in our case at least the leak will be in the specific tissue. The probable consequences of the leak will be studied. Secondly, the feeding of domestic birds with the doxycycline antibiotic can compromise its safety as biological products. However it is a temporary measure that 
could help to save the stock. The efficacy of our strategy needs to be evaluated in vivo to resolve all the concerns.'

Competing Interests: No competing interests were disclosed.

Reviewer Report 12 March 2018

https://doi.org/10.5256/f1000research.14654.r31110

(c) 2018 Air G. This is an open access peer review report distributed under the terms of the Creative Commons Attribution License, which permits unrestricted use, distribution, and reproduction in any medium, provided the original work is properly cited.

\section{Gillian M Air}

Department of Biochemistry and Molecular Biology, University of Oklahoma Health Sciences Center, Oklahoma City, OK, USA

The authors have expressed membrane-anchored constructs of influenza, human and bacterial sialidases in cells and measured the effect on cell surface sialylated glycans. The study is preliminary but well executed as far as it goes.

General comments:

1. The manuscript does not include evaluation of influenza virus infection so the title is exaggerated. Maybe "Evaluation of potential defense strategy...". would be less misleading.

2. The Figure legends are very terse and a reader needs to make some guesses at what is being shown. For example, what are the boxes on the panels in Figures 3 and 4?

3. The manuscript needs to be checked for spelling. "sialilation" appears several times and there are other errors such as S. typhimyrium on p.7.

Specific comments:

1. Abstract: I have not seen data that shows the H5N1 virus is increasingly infective for humans. Either reference this statement or modify it.

2. Abstract and text: the increased activity seen with influenza neuraminidase is likely due to the construct being more native and better folded. The $S$. typhimurium sialidase itself has much higher activity than influenza NA.

3. P.5: the Maakia amurensis lectins have been variable between suppliers and one form binds additional glycans, as has been discussed in several recent publications. More information is needed to be sure the lectin used has alpha2-3 specificity. 
4. P.5, first paragraph of Results. "The optimal sialic acids level on the cells surface". I think this should refer to sialidase, not sialic acid.

5. P.5 The influenza NA does indeed cleave alpha2- 6 linked sialic acid but at $1 / 3$ to $1 / 5$ the rate of alpha2-3.

6. P.5: while the assay to show reduction of cell surface sialic acids fits the goal of the project, it might be useful to carry out classical enzyme assays using the expressed sialidases to determine if the constructs used have similar activity to their wild type counterparts and if not, if they can be improved.

Is the work clearly and accurately presented and does it cite the current literature?

Yes

Is the study design appropriate and is the work technically sound?

Partly

Are sufficient details of methods and analysis provided to allow replication by others? Yes

If applicable, is the statistical analysis and its interpretation appropriate?

Yes

Are all the source data underlying the results available to ensure full reproducibility? Yes

Are the conclusions drawn adequately supported by the results?

Partly

Competing Interests: No competing interests were disclosed.

Reviewer Expertise: Influenza

I confirm that I have read this submission and believe that I have an appropriate level of expertise to confirm that it is of an acceptable scientific standard, however I have significant reservations, as outlined above.

Author Response 03 May 2018

Ekaterina Antonova, Moscow Institute of Physics and Technology, Dolgoprudny, Russian Federation

Respond to the general comments:

1.The correction has been done in the version 2 .

2.Figure legends have been supplemented with details in the version 2 .

3. The manuscript has been checked for spelling and found errors and typos were corrected in the version 2.

Respond to the specific comments:

1.The statement was modified to 'Avian $\mathrm{H} 5 \mathrm{~N} 1$ and $\mathrm{H} 7 \mathrm{~N} 9$ virus has a potential pandemic 
threat' in the version 2 .

2.The information was added in the Results/Transient expression of genetic constructs in HEK293 cell lines.

3.Maakia amurensis lectins from bioWORLD that have declared alpha(2;3) specificity were used as analogous to an avian hemagglutinin due to they share similar structural fold.

The lectins were used for preliminary study and it is planned to continue the research with an avian virus.

4. Both statements make sense, however "the optimal sialic acids level" is the result of sialidases expression and it undermines not only the level of sialidases on the cell surface but their catalytic activity.

5.The statement «Unlike human HA that preferentially recognises a (2-6), the viral NA of H1N1 has cleavage activity to both (2-6) and a(2-3) types of linkages» was supplemented with the information that NA also cleaves alpha2-6 linked sialic acids at lower rate than alpha2-3.

6.The aim of the project was to compare created genetic constructs between each other and use some of them in future researches. We didn't try to achieve similar activity of the constructs to their recombinant counterparts. However it would be interesting to check how folding and localisation influence on the enzymes activity.

Competing Interests: No competing interests were disclosed.

The benefits of publishing with F1000Research:

- Your article is published within days, with no editorial bias

- You can publish traditional articles, null/negative results, case reports, data notes and more

- The peer review process is transparent and collaborative

- Your article is indexed in PubMed after passing peer review

- Dedicated customer support at every stage

For pre-submission enquiries, contact research@f1000.com 